\title{
Orthoptic Sets and Quadric Hypersurfaces
}

\author{
François Dubeau ${ }^{1 *}$
}

\begin{abstract}
Orthoptic curves for the conics are well known. It is the Monge's circle for ellipse and hyperbola, and for parabola it is its directrix. These conics are level sets of quadratic functions in the plane. We consider level sets of quadratic functions in higher dimension, known as quadric hypersurfaces. For these hypersurfaces we present and study their orthoptic sets, which extend the idea of orthoptic curves for conics.
\end{abstract}

Keywords: Directrix, Monge's circle, orthoptic set, quadric hypersurface

2010 AMS: Primary 51M05, Secondary 53A05, 15 A63

${ }^{1}$ Département de Mathématiques, Faculté des sciences, Université de Sherbrooke, Sherbrooke (Qc), Canada, ORCID: 0000000229563208

*Corresponding author: francois.dubeau@usherbrooke.ca

Received: 15 April 2021, Accepted: 1 October 2021, Available online: 1 October 2021

\section{Introduction}

In the plane the orthoptic curve is the locus of the points by which pass two perpendicular tangents to the curve, in other words, the locus of the points from which we "see" the curve under a right angle. For the conics in the plane it is related to Monge's work [3].

For ellipse and hyperbola it is called the Monge's circle. Given the ellipse $\frac{x^{2}}{a^{2}}+\frac{y^{2}}{b^{2}}=1$, the Monge's circle is $x^{2}+y^{2}=a^{2}+b^{2}$, while for the hyperbola $\frac{x^{2}}{a^{2}}-\frac{y^{2}}{b^{2}}=1$, it is $x^{2}+y^{2}=a^{2}-b^{2}$, which exists only for $a^{2}-b^{2}>0$. For the parabola $y^{2}=2 p x$, the orthoptic curve is its directrix $x=-p / 2$. See for example [1], [2], [4] for more details.

For these examples in the plane we need two perpendicular tangents to a curve. So the two normal vectors to the tangent planes, which are also normal vectors to the curve, are also orthogonal. One way to consider this locus in higher dimension is to consider a set of tangent planes to the hypersurface such that the set of their normal vectors, to the given tangent planes, form an orthogonal set.

In this paper we consider a natural way to define an orthoptic set associated to a quadric hypersurface. We first present, in Section 2, the surface we are considering and define what we will consider as an orthoptic set. Then some notations are introduced in Section 3. The next two sections contain the presentation and the proofs of our main results. In Section 4 we consider ellipsoid and hyperboloid hypersurfaces. For ellipsoid, the technique in $\mathbb{R}^{3}$ seems to be due to Monge, as reported in [5] where it is referred to [3]. We present here that it can be extended not only to ellipsoid in $\mathbb{R}^{n}$, but also to hyperboloid in $\mathbb{R}^{n}$. Moreover in Section 5 a variant of this technique is also used to determine the orthoptic set for paraboloid hypersurfaces. In the last section, the conclusion, a summary is presented and some questions are raised for future research.

The contribution of this paper is to present results for orthoptic sets, not only for conics in $\mathbb{R}^{2}$ [4] and quadrics in $\mathbb{R}^{3}$ [5], but also for quadric hypersurfaces in $\mathbb{R}^{n}$. Even thought it can be said that the technique for ellipsoid in $\mathbb{R}^{3}$ can be extended to higher dimension [5], we present this extension not only for ellipsoids, but also for hyperboloids and paraboloids. We will see that it is a nice application of the trace operator of a matrix. Finally, one question remains unanswered. The results say that the orthoptic sets are included in some sets, but are these sets exactly the orthoptic sets. This result is true in $\mathbb{R}^{n}$ for $n=2,3$, but for $n>3$ it is an open question. 


\section{Preliminaries}

\subsection{Quadric hypersurfaces}

The two quadratic functions we will study lead to ellipsoid or hyperboloid hypersurface defined by

$$
f(x, y)=\sum_{i=1}^{I} \frac{x_{i}^{2}}{a_{i}^{2}}-\sum_{j=1}^{J} \frac{y_{j}^{2}}{b_{j}^{2}}=1
$$

for $(x, y) \in \mathbb{R}^{I+J}$, and to paraboloid surface defined by

$$
g(x, y, z)=\sum_{i=1}^{I} \frac{x_{i}^{2}}{a_{i}^{2}}-\sum_{j=1}^{J} \frac{y_{j}^{2}}{b_{j}^{2}}-\sum_{k=1}^{K} p_{k} z_{k}=0,
$$

for $(x, y, z) \in \mathbb{R}^{I+J+K}$.

\subsection{Orthoptic surface}

Based on the fact that in the plane each point of the orthoptic curve is associated to two normal vectors to the tangent planes or also to the curve, the next definition is suggested for a generalization in multidimensional Euclidean spaces of the usual orthoptic curve in the plane.

Definition. Let a hypersurface $\mathscr{S}$ defined by $h(\xi)=0$ in $\mathbb{R}^{L}$. The orthoptic set is the set of points common to L tangent planes to $\mathscr{S}$ under the condition that the L normals to the tangent planes form an orthogonal set.

\section{Notations}

Let $x=\left(x_{1}, \ldots, x_{I}\right) \in \mathbb{R}^{I}, y=\left(y_{1}, \ldots, y_{J}\right) \in \mathbb{R}^{J}, z=\left(z_{1}, \ldots, z_{K}\right)$ and $p=\left(p_{1}, \ldots, p_{K}\right) \in \mathbb{R}^{K}$. Let $N=I+J$ and $M=N+K=$ $I+J+K$. Let us introduce the $I$ 'th order diagonal matrix $A=\operatorname{diag}\left(a_{i}\right)$, the $J$ 'th order diagonal matrix $B=\operatorname{diag}\left(b_{j}\right)$, and the $N$ 'th order diagonal matrix

$$
P=\left[\begin{array}{cc}
A & O \\
O & \imath B
\end{array}\right]
$$

where $l$ is the unit complex number such that $\iota^{2}=-1$. For any integer $l \in \mathbb{Z}$, we have

$$
A^{l}=\operatorname{diag}\left(a_{i}^{l}\right) \quad \text { and } \quad B^{l}=\operatorname{diag}\left(b_{j}^{l}\right)
$$

and also

$$
P^{l}=\left[\begin{array}{cc}
A^{l} & O \\
O & l^{l} B^{l}
\end{array}\right]
$$

For any (line vector) $q \in \mathbb{R}^{L}, q^{t}$ will be its (column vector) transpose. So, we can rewrite the quadratic form $f(x, y)$ as

$$
f(x, y)=x A^{-2} x^{t}-y B^{-2} y^{t}=v P^{-2} v^{t}=f(v),
$$

where $v=(x, y) \in \mathbb{R}^{N}$, and the quadratic form $g(x, y, z)$ as

$$
g(x, y, z)=x A^{-2} x^{t}-y B^{-2} y^{t}-2 p z^{t}=v P^{-2} v^{t}-2 p z^{t}=g(w),
$$

where $w=(v, z)=(x, y, z) \in \mathbb{R}^{M}$.

\section{Ellipsoid and Hyperboloid hypersurfaces}




\subsection{Tangent planes}

For

$$
f(v)=v P^{-2} v^{t},
$$

a row normal vector to the surface $f(v)=1$ at a point $v_{0}$ of this surface, noted $V\left(v_{0}\right)$, can be taken to be

$$
V\left(v_{0}\right)=\frac{1}{2} \nabla f\left(v_{0}\right)=v_{0} P^{-2} .
$$

The tangent plane to $f(v)=1$ at $v_{0}$ is given by the condition

$$
V\left(v_{0}\right)\left(v-v_{0}\right)^{t}=0
$$

which gives

$$
V\left(v_{0}\right) v^{t}=V\left(v_{0}\right) v_{0}^{t}=v_{0} P^{-2} v_{0}^{t}=f\left(v_{0}\right)=1 .
$$

\subsection{Orthoptic set}

Let us suppose that there exists a finite sequence of points $\left\{v_{n}\right\}_{n=1}^{N}$ such that $f\left(v_{n}\right)=1$ for $n=1, \ldots, N$, and $\left\{V\left(v_{n}\right)\right\}_{n=1}^{N}$ is an orthogonal set. Let us look for the common point to the $N$ tangent planes to the surface $f\left(v_{n}\right)=1$ at $v_{n}$, that is to say a point $\widetilde{v}=(\widetilde{x}, \widetilde{y})$ such that

$$
V\left(v_{n}\right) \widetilde{v}^{t}=1
$$

for $n=1, \ldots, N$. We have to solve the linear system

$$
\left[\begin{array}{c}
V\left(v_{1}\right) \\
\vdots \\
V\left(v_{N}\right)
\end{array}\right] \widetilde{v}^{t}=\left[\begin{array}{c}
1 \\
\vdots \\
1
\end{array}\right]
$$

Using the orthogonality property of the family of normal vectors, we get

$$
\left[\begin{array}{c}
V\left(v_{1}\right) \\
\vdots \\
V\left(v_{N}\right)
\end{array}\right]^{-1}=\left[\begin{array}{lll}
\frac{V^{t}\left(v_{1}\right)}{\left|V\left(v_{1}\right)\right|^{2}} & \cdots & \frac{V^{t}\left(v_{N}\right)}{\left|V\left(v_{N}\right)\right|^{2}}
\end{array}\right]
$$

and then

$$
\widetilde{v}=\sum_{n=1}^{N} \frac{1}{\left|V\left(v_{n}\right)\right|^{2}} V\left(v_{n}\right) .
$$

Again, from the orthogonality condition we get

$$
|\widetilde{v}|^{2}=\widetilde{v} \widetilde{v}^{t}=\sum_{n=1}^{N} \frac{1}{\left|V\left(v_{n}\right)\right|^{4}} V\left(v_{n}\right) V^{t}\left(v_{n}\right)=\sum_{n=1}^{N} \frac{1}{\left|V\left(v_{n}\right)\right|^{2}} .
$$

Let us look at the inverse. We have

$$
I=\left[\begin{array}{c}
V\left(v_{1}\right) \\
\vdots \\
V\left(v_{N}\right)
\end{array}\right]\left[\begin{array}{lll}
\frac{V^{t}\left(v_{1}\right)}{\left|V\left(v_{1}\right)\right|^{2}} & \cdots & \frac{V^{t}\left(v_{N}\right)}{\left|V\left(v_{N}\right)\right|^{2}}
\end{array}\right]
$$

and also

$$
\begin{aligned}
I & =\left[\begin{array}{lll}
\frac{V^{t}\left(v_{1}\right)}{\left|V\left(v_{1}\right)\right|^{2}} & \cdots & \frac{V^{t}\left(v_{N}\right)}{\left|V\left(v_{N}\right)\right|^{2}}
\end{array}\right]\left[\begin{array}{c}
V\left(v_{1}\right) \\
\vdots \\
V\left(v_{N}\right)
\end{array}\right] \\
& =\sum_{n=1}^{N} \frac{1}{\left|V\left(v_{l}\right)\right|^{2}} V^{t}\left(v_{n}\right) V\left(v_{n}\right) \\
& =\sum_{n=1}^{N} \frac{1}{\left|V\left(v_{l}\right)\right|^{2}} P^{-2} v_{n}^{t} v_{n} P^{-2} .
\end{aligned}
$$


Let us observe that

$$
P^{2}=P I P=\sum_{n=1}^{N} \frac{1}{\left|V\left(v_{n}\right)\right|^{2}} P^{-1} v_{n}^{t} v_{n} P^{-1},
$$

and taking the trace on both sides, we get

$$
\operatorname{Trace}\left(P^{2}\right)=\sum_{i=1}^{I} a_{i}^{2}-\sum_{j=1}^{J} b_{j}^{2}
$$

and

$$
\begin{aligned}
\operatorname{Trace}\left(P^{2}\right) & =\sum_{n=1}^{N} \frac{1}{\left|V\left(v_{n}\right)\right|^{2}} \operatorname{Trace}\left(P^{-1} v_{n}^{t} v_{n} P^{-1}\right) \\
& =\sum_{n=1}^{N} \frac{1}{\left|V\left(v_{n}\right)\right|^{2}} \operatorname{Trace}\left(v_{n} P^{-2} v_{n}^{t}\right) \\
& =\sum_{n=1}^{N} \frac{1}{\left|V\left(v_{n}\right)\right|^{2}} f\left(v_{n}\right) \\
& =\sum_{n=1}^{N} \frac{1}{\left|V\left(v_{n}\right)\right|^{2}},
\end{aligned}
$$

where we used the fact that $\operatorname{Trace}\left(H H^{t}\right)=\operatorname{Trace}\left(H^{t} H\right)$. So we obtain the result we were looking for.

Theorem 4.1. Let the hypersurface, ellipsoid or hyperboloid, be defined by

$$
\sum_{i=1}^{I} \frac{x_{i}^{2}}{a_{i}^{2}}-\sum_{j=1}^{J} \frac{y_{j}^{2}}{b_{j}^{2}}=1,
$$

in $\mathbb{R}^{N}$ where $N=I+J$. The orthoptic set of this hypersurface, if it exists, is included in the hypersphere of radius $\sqrt{\sum_{i=1}^{I} a_{i}^{2}-\sum_{j=1}^{J} b_{j}^{2}} \geq 0$ given by

$$
\sum_{i=1}^{I} x_{i}^{2}+\sum_{j=1}^{J} y_{j}^{2}=\sum_{i=1}^{I} a_{i}^{2}-\sum_{j=1}^{J} b_{j}^{2}
$$

\section{Paraboloid hypersurface}

\subsection{Tangent planes}

For

$$
g(w)=v P^{-2} v^{t}-2 p z^{t},
$$

a row normal vector to the surface $g(w)=0$ at a point $w_{0}$ of this surface, noted $W\left(w_{0}\right)$, can be taken to be

$$
W\left(w_{0}\right)=\frac{1}{2} \nabla g\left(w_{0}\right)=\left(v_{0} P^{-2},-p\right) .
$$

The tangent plane to $g(w)=0$ at $w_{0}$ is given by the condition

$$
W\left(w_{0}\right)\left(w-w_{0}\right)^{t}=0,
$$

which gives

$$
W\left(w_{0}\right) w^{t}=W\left(w_{0}\right) w_{0}^{t}=v_{0} P^{-2} v_{0}^{t}-p z_{0}^{t}=g\left(w_{0}\right)+p z_{0}^{t}=p z_{0}^{t} .
$$




\subsection{Orthoptic set}

Let us suppose that there exists a sequence of points $\left\{w_{m}\right\}_{m=1}^{M}$ such that $g\left(w_{m}\right)=0$ for $m=1, \ldots, M$, and $\left\{W\left(w_{m}\right)\right\}_{m=1}^{M}$ is an orthogonal sequence. Let us look for the common point to the $M$ tangent planes to the surface $g\left(w_{m}\right)=0$ at $w_{m}$, that is to say a point $\widetilde{w}=(\widetilde{x}, \widetilde{y}, \widetilde{z})$ such that

$$
W\left(w_{m}\right) \widetilde{w}^{t}=p z_{m}^{t}
$$

for $m=1, \ldots, M$. We have to solve the linear system

$$
\left[\begin{array}{c}
W\left(w_{1}\right) \\
\vdots \\
W\left(w_{M}\right)
\end{array}\right] \widetilde{w}^{t}=\left[\begin{array}{c}
p z_{1}^{t} \\
\vdots \\
p z_{M}^{t}
\end{array}\right] .
$$

Using the orthogonality properties of the family of normal vectors, we get

$$
\left[\begin{array}{c}
W\left(w_{1}\right) \\
\vdots \\
W\left(w_{M}\right)
\end{array}\right]^{-1}=\left[\begin{array}{lll}
\frac{W^{t}\left(w_{1}\right)}{\left|W\left(w_{1}\right)\right|^{2}} & \cdots & \frac{W^{t}\left(w_{M}\right)}{\left|W\left(w_{M}\right)\right|^{2}}
\end{array}\right]
$$

and then

$$
\widetilde{w}^{t}=\sum_{m=1}^{M} \frac{1}{\left|W\left(w_{m}\right)\right|^{2}} W^{t}\left(w_{m}\right) p z_{m}^{t}
$$

and so

$$
p \widetilde{z}^{t}=-|p|^{2} \sum_{m=1}^{M} \frac{1}{\left|W\left(w_{m}\right)\right|^{2}} p z_{m}^{t}
$$

Let us look at the inverse. We have

$$
I=\left[\begin{array}{c}
W\left(w_{1}\right) \\
\vdots \\
W\left(w_{M}\right)
\end{array}\right]\left[\begin{array}{lll}
\frac{W^{t}\left(w_{1}\right)}{\left|W\left(w_{1}\right)\right|^{2}} & \cdots & \frac{W^{t}\left(w_{M}\right)}{\left|W\left(w_{M}\right)\right|^{2}}
\end{array}\right]
$$

and also

$$
\begin{aligned}
I & =\left[\begin{array}{lll}
\frac{W^{t}\left(w_{1}\right)}{\left|W\left(w_{1}\right)\right|^{2}} & \cdots & \frac{W^{t}\left(w_{M}\right)}{\left|W\left(w_{M}\right)\right|^{2}}
\end{array}\right]\left[\begin{array}{c}
W\left(w_{1}\right) \\
\vdots \\
W\left(w_{M}\right)
\end{array}\right] \\
& =\sum_{m=1}^{M} \frac{1}{\left|W\left(w_{l}\right)\right|^{2}} W^{t}\left(w_{m}\right) W\left(w_{m}\right) \\
& =\sum_{m=1}^{M} \frac{1}{\left|W\left(w_{m}\right)\right|^{2}}\left[\begin{array}{cc}
P^{-2} v_{l}^{t} v_{l} P^{-2} & P^{-2} v_{l}^{t} p \\
p_{t} v_{l} P^{-2} & p^{t} p
\end{array}\right] .
\end{aligned}
$$

Let us first observe that

$$
\begin{aligned}
|p|^{2} & =\left[\begin{array}{ll}
0 & p
\end{array}\right] I\left[\begin{array}{c}
0 \\
p^{t}
\end{array}\right] \\
& =\sum_{m=1}^{M} \frac{1}{\left|W\left(w_{m}\right)\right|^{2}}\left[\begin{array}{ll}
0 & p
\end{array}\right]\left[\begin{array}{cc}
P^{-2} v_{l}^{t} v_{l} P^{-2} & P^{-2} v_{l}^{t} p \\
p_{t} v_{l} P^{-2} & p^{t} p
\end{array}\right]\left[\begin{array}{c}
0 \\
p^{t}
\end{array}\right] \\
& =\sum_{m=1}^{M} \frac{1}{\left|W\left(w_{m}\right)\right|^{2}} p p^{t} p p^{t} \\
& =\sum_{m=1}^{M} \frac{1}{\left|W\left(w_{m}\right)\right|^{2}}|p|^{4},
\end{aligned}
$$

so

$$
|p|^{2} \sum_{m=1}^{M} \frac{1}{\left|W\left(w_{m}\right)\right|^{2}}=1
$$


Using any $K^{\prime}$ th order diagonal matrix $Q=\operatorname{diag}\left(q_{k}\right)$ where $q_{k} \in \mathbb{R}$ for $k=1, \ldots, K$, we have

$$
\begin{aligned}
{\left[\begin{array}{cc}
P^{2} & 0 \\
0 & Q^{2}
\end{array}\right] } & =\left[\begin{array}{ll}
P & 0 \\
0 & Q
\end{array}\right] I\left[\begin{array}{cc}
P & 0 \\
0 & Q
\end{array}\right] \\
& =\sum_{m=1}^{M} \frac{1}{\left|W\left(w_{m}\right)\right|^{2}}\left[\begin{array}{cc}
P^{-1} v_{l}^{t} v_{l} P^{-1} & P^{-1} v_{l}^{t} p Q \\
Q p^{t} v_{l} P^{-1} & Q p^{t} p Q
\end{array}\right],
\end{aligned}
$$

and taking the trace on both sides, we get

$$
\operatorname{Trace}\left(P^{2}\right)+\operatorname{Trace}\left(Q^{2}\right)=\sum_{i=1}^{I} a_{i}^{2}-\sum_{j=1}^{J} b_{j}^{2}+\sum_{k=1}^{K} q_{k}^{2},
$$

and

$$
\begin{aligned}
& \sum_{m=1}^{M} \frac{1}{\left|W\left(w_{m}\right)\right|^{2}} \operatorname{Trace}\left[\begin{array}{cc}
P^{-1} v_{l}^{t} v_{l} P^{-1} & P^{-1} v_{l}^{t} p Q \\
Q p^{t} v_{l} P^{-1} & Q p^{t} p Q
\end{array}\right] \\
& \quad=\sum_{m=1}^{M} \frac{1}{\left|W\left(w_{m}\right)\right|^{2}}\left[\operatorname{Trace}\left(P^{-1} v_{m}^{t} v_{m} P^{-1}\right)+\operatorname{Trace}\left(Q p^{t} p Q\right)\right] \\
& \quad=\sum_{m=1}^{M} \frac{1}{\left|W\left(w_{m}\right)\right|^{2}}\left[\operatorname{Trace}\left(v_{m} P^{-2} v_{m}^{t}\right)+\operatorname{Trace}\left(p Q^{2} p^{t}\right)\right] \\
& \quad=\sum_{m=1}^{M} \frac{1}{\left|W\left(w_{m}\right)\right|^{2}}\left[v_{m} P^{-2} v_{m}^{t}+p Q^{2} p^{t}\right] \\
& =\sum_{m=1}^{M} \frac{1}{\left|W\left(w_{m}\right)\right|^{2}}\left[P\left(w_{m}\right)+2 p z_{m}^{t}+p Q^{2} p^{t}\right] \\
& =2 \sum_{m=1}^{M} \frac{1}{\left|W\left(w_{m}\right)\right|^{2}} p z_{m}^{t}+p Q^{2} p^{t} \sum_{m=1}^{M} \frac{1}{\left|W\left(w_{m}\right)\right|^{2}}
\end{aligned}
$$

For $Q=0$ we obtain

$$
\sum_{i=1}^{I} a_{i}^{2}-\sum_{j=1}^{J} b_{j}^{2}=2 \sum_{m=1}^{M} \frac{1}{\left|W\left(w_{m}\right)\right|^{2}} p z_{m}^{t},
$$

and for $Q=I$, since $\operatorname{Trace}\left(Q^{2}\right)=\operatorname{Trace}(I)=K$ and $p Q^{2} p^{t}=p p^{t}=|p|^{2}$, we get

$$
\begin{aligned}
\sum_{i=1}^{I} a_{i}^{2}-\sum_{j=1}^{J} b_{j}^{2}+K & =2 \sum_{m=1}^{M} \frac{1}{\left|W\left(w_{m}\right)\right|^{2}} p z_{m}^{t}+|p|^{2} \sum_{m=1}^{M} \frac{1}{\left|W\left(w_{m}\right)\right|^{2}} \\
& =2 \sum_{m=1}^{M} \frac{1}{\left|W\left(w_{m}\right)\right|^{2}} p z_{m}^{t}+1 .
\end{aligned}
$$

This is possible only for $K=1$. So we obtain the result we were looking for.

Theorem 5.1. Let the hypersurface, a paraboloid, defined by

$$
\sum_{i=1}^{I} \frac{x_{i}^{2}}{a_{i}^{2}}-\sum_{j=1}^{J} \frac{y_{j}^{2}}{b_{j}^{2}}-\sum_{k=1}^{K} p_{k} z_{k}=0,
$$

in $\mathbb{R}^{M}$ where $M=N+K=I+J+K$.

For $K=1$, the orthoptic set might exist and, if it exists, is included in the hyperplane

$$
z=-\frac{p}{2}\left[\sum_{i=1}^{I} a_{i}^{2}-\sum_{j=1}^{J} b_{j}^{2}\right],
$$

where we have considered $p>0$.

For $K>1$ the orthoptic set does not exist. 
Let us observe that the fact that $K=1$ in this last theorem is not a surprise. Indeed for $K>1$, since the last $K$ entries of any normal vectors are all equal to 1 , it is not possible to find a set of $M=I+J+K$ orthogonal (normal) vectors to the paraboloid as assumed to get the result.

\section{Conclusion}

We have introduced orthoptic sets for hypersurfaces associated to quadratic forms in $\mathbb{R}^{n}$. At least one interesting question remains: are the hypersphere in Theorem 4.1 or the hyperplane in Theorem 5.1 exactly the orthoptic surfaces ? In other words, to any point on the given hypersphere or hyperplane does there exists a set of orthogonal normals for which the point is the unique common point to the corresponding set of planes? As an example, for Theorem 4.1 with $N=2$ and $I=1=J$, if the radius is 0 , which means that $a_{1}=b_{1}$, it is not possible to find a set of 2 orthogonal normals, except if we consider that the two asymptotes are tangent at infinity to the hyperbola. So what happens in higher dimension?

\section{Acknowledgement}

The authors would like to express their sincere thanks to the editor and the anonymous reviewers for their helpful comments and suggestions.

\section{Funding}

This work has been financially supported by an individual discovery grant from NSERC (Natural Sciences and Engineering Research Council of Canada).

\section{Availability of data and materials}

Not applicable.

\section{Competing interests}

The authors declare that they have no competing interests.

\section{Author's contributions}

All authors contributed equally to the writing of this paper. All authors read and approved the final manuscript.

\section{References}

[1] I. Assem and J.C. Bustamante (2017). Géométrie analytique, Presses internationales Polytechnique, Montréal.

[2] Y. Ladegaillerie, Géometrie affine, projective, euclidienne et anallagmatique, Ellipses Édition Marketing S.A., Paris, 2003.

[3] O.J. Staude, Flächen 2. Ordnung und ihre Systeme und Durchdringungskurven. Encyklopädie der math., Wiss.III.2.1, no. C2, 161-256, B.G. Teubner, Leipzig, 2015.

[4] G. Glaeser, H. Stachel, B. Odehnal, The Universe of Conics, Springer Spektrum, Springer-Verlag Berlin Heidelberg, 2016.

[5] B. Odehnal, H. Stachel, G. Glaeser, The Universe of Quadrics, Springer Spektrum, Springer-Verlag Berlin Heidelberg, 2020 . 